\title{
DIGITALCOMMONS
}

@WAYNESTATE —

Wayne State University

Physics and Astronomy Faculty Research

Publications

Physics and Astronomy

$4-1-1990$

\section{Rovibrationally enhanced dissociative electron attachment to molecular lithium}

J M. Wadehra

Wayne State University, ad5541@wayne.edu

\section{Recommended Citation}

Wadehra JM. Rovibrationally enhanced dissociative electron attachment to molecular lithium. Phys. Rev. A. 1990;41(7):3607-3611. doi: 10.1103/PhysRevA.41.3607

Available at: http://digitalcommons.wayne.edu/phy_astro_frp/96

This Article is brought to you for free and open access by the Physics and Astronomy at DigitalCommons@WayneState. It has been accepted for inclusion in Physics and Astronomy Faculty Research Publications by an authorized administrator of DigitalCommons@WayneState. 


\title{
Rovibrationally enhanced dissociative electron attachment to molecular lithium
}

\author{
J. M. Wadehra \\ Department of Physics and Astronomy, Wayne State University, Detroit, Michigan 48202* \\ and Wright Research and Development Center, Wright-Patterson Air Force Base, Ohio 45433
}

(Received 23 August 1989)

\begin{abstract}
We have investigated the role played by initial rovibrational excitation of $\mathrm{Li}_{2}$ on the cross sections and rates for dissociative electron attachment to the molecule. For a given internal energy, the vibrational excitation enhances the attachment cross section more than the rotational excitation. The attachment cross sections and the attachment rates reach their maximum values when the process of dissociative attachment to rovibrationally excited molecules is still endoergic and, furthermore, these quantities stay close to their maximum values even when the process changes from being endoergic to exoergic. The upper bounds on the cross sections and the rates for dissociative electron attachment to $\mathrm{Li}_{2}$ are $12.8 \AA^{2}$ and $1.25 \times 10^{-8} \mathrm{~cm}^{3} \mathrm{~s}^{-1}$. At a fixed electron temperature, the kinetic energy of the negative ion formed by this process increases as the vibrational quantum number of the initial neutral molecule increases; the maximum kinetic energy of the $\mathrm{Li}^{-}$ion formed by attachment to the $v=12$ level of $\mathrm{Li}_{2}$ is $0.153 \mathrm{eV}$.
\end{abstract}

\section{INTRODUCTION}

It has been amply demonstrated, both theoretically ${ }^{1}$ as well as experimentally, ${ }^{2}$ that the cross sections for $\mathrm{H}^{-}$ formation via the process of dissociative electron attachment to molecular hydrogen are significantly enhanced if the molecule $\mathrm{H}_{2}$ is initially rovibrationally excited. In order to assess the analogous degree of enhancement for other molecular systems, we have presently investigated in detail the effect of initial rovibrational excitation on the rate of production of $\mathrm{Li}^{-}$via the process of dissociative electron attachment to diatomic lithium molecules. Investigations of electron attachment to lithium molecules are especially appropriate at this time due to several reasons. First, recent experimental observations ${ }^{3}$ reveal that the rate of $\mathrm{Li}^{-}$formation by the impact of thermal electrons on highly vibrationally excited $\mathrm{Li}_{2}$ is $(2 \pm 1) \times 10^{-8} \mathrm{~cm}^{3} \mathrm{~s}^{-1}$. One of the principal aims of the present work is to confirm this experimental observation of attachment rate by explicit calculations as well as to provide detailed cross sections for electron attachment to $\mathrm{Li}_{2}$. Second, the $\mathrm{Li}^{-}$ions could possibly play, in the future, the same roles as have been played by $\mathrm{H}^{-}$ions for neutral beam formation. Third, since lithium dimers are isovalent with $\mathrm{H}_{2}$, an investigation of the dependence of the electron attachment to $\mathrm{Li}_{2}$ on the initial rovibrational excitation of the molecule would be similar to the previous detailed study ${ }^{4}$ on $\mathrm{H}_{2}$.

Similarities between $\mathrm{Li}_{2}$ and $\mathrm{H}_{2}$ suggest that theoretical methods used successfully in the past for obtaining the cross sections and rates of electron attachment to $\mathrm{H}_{2}$ can be employed for analogous investigations for $\mathrm{Li}_{2}$. In particular, the process of dissociative electron attachment to $\mathrm{Li}_{2}$ is understood to proceed through the formation of an intermediate resonant anion state $\mathrm{Li}_{2}{ }^{-}$which, on dissociation, leads to $\mathrm{Li}^{-}$. The fact that both the lithium dimer molecules and the hydrogen molecules are isovalent pro- vides similarities between the electronic configurations of the two molecules. For example, the lowest electronic states of the negative molecular ions with configurations $\left(1 \sigma_{g}\right)^{2}\left(1 \sigma_{u}\right)^{2}\left(2 \sigma_{g}\right)^{2}\left(2 \sigma_{u}\right)$ for $\mathrm{Li}_{2}{ }^{-}$and $\left(1 \sigma_{g}\right)^{2}\left(1 \sigma_{u}\right)$ for $\mathrm{H}_{2}{ }^{-}$have similar symmetry, namely, ${ }^{2} \Sigma_{u}^{+}$. However, compared to the hydrogen molecule, the lithium molecule possesses a large polarizability and a weak bond strength which makes the ground state of $\mathrm{Li}_{2}{ }^{-}$a true bound state. In the case of $\mathrm{H}_{2}{ }^{-}$, on the other hand, the ${ }^{2} \Sigma_{u}^{+}$state is a true bound state only for internuclear separations $R$ larger than 2.9 a.u. and is an autodetaching state for smaller values of $R$. The first excited state of the negative molecular ions with symmetry ${ }^{2} \Sigma_{g}^{+}$and configurations $\left(1 \sigma_{g}\right)^{2}\left(1 \sigma_{u}\right)^{2}\left(2 \sigma_{g}\right)\left(2 \sigma_{u}\right)^{2}$ for $\mathrm{Li}_{2}{ }^{-}$and $\left(1 \sigma_{g}\right)\left(1 \sigma_{u}\right)^{2}$ for $\mathrm{H}_{2}{ }^{-}$is partly Feshbach and partly shape resonance in nature for both. This state is the essential channel for dissociative attachment of low-energy electrons to lithium molecules. The process of dissociative electron attachment to molecular lithium, then, is:

$$
e^{-}+\mathrm{Li}_{2}\left(X^{1} \Sigma_{g}^{+}\right) \rightarrow \mathrm{Li}_{2}^{-}\left(A^{2} \Sigma_{g}^{+}\right) \rightarrow \mathrm{Li}+\mathrm{Li}^{-} .
$$

\section{iI. Calculations}

Fortunately, a number of accurate calculations ${ }^{5-9}$ of the potential curves of the $X^{1} \Sigma_{g}^{+}$state of $\mathrm{Li}_{2}$ and the $A^{2} \Sigma_{g}^{+}$state of $\mathrm{Li}_{2}{ }^{-}$are available. The potential curves that we utilize in the present work were obtained ${ }^{9}$ by an $a b$ initio calculation using optimized configurationinteraction (CI) wave functions built from orthonormal Slater-type orbitals (STO's). Because of its nature (namely Feshbach) the ${ }^{2} \Sigma_{g}^{+}$resonance of $\mathrm{Li}_{2}{ }^{-}$is expected to have a small width and a long lifetime. Potential curves of the electronic states of $\mathrm{Li}_{2}$ and $\mathrm{Li}_{2}{ }^{-}$relevant to the attachment process are shown in Fig. 1. The $A^{2} \Sigma_{g}^{+}$electronic state of $\mathrm{Li}_{2}{ }^{-}$exhibits, due to its autodetaching nature, a complex potential-energy curve whose real part 


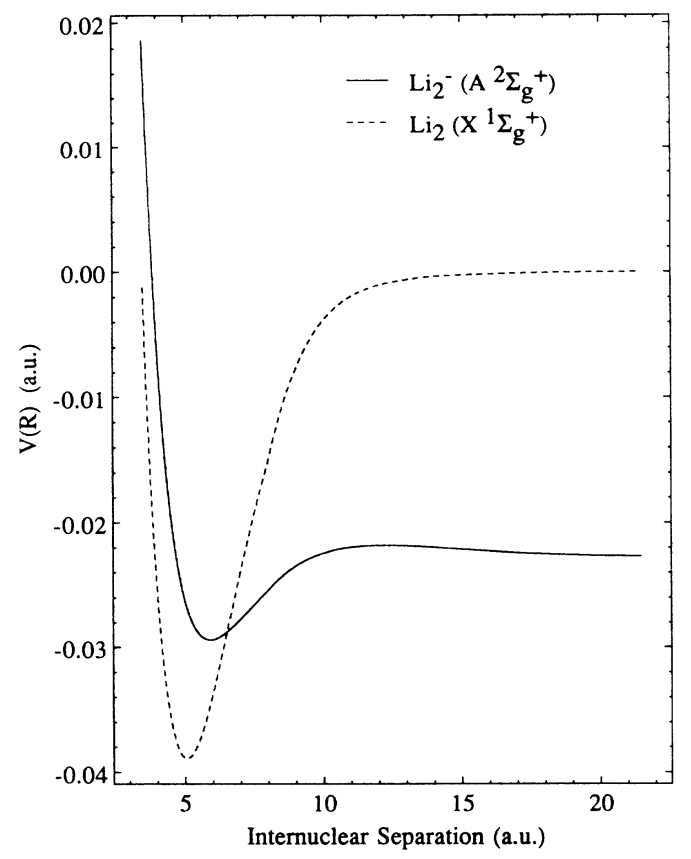

FIG. 1. The potential curves of $\mathrm{Li}_{2}\left(X^{1} \Sigma_{g}^{+}\right)$and $\mathrm{Li}_{2}{ }^{-}\left(A^{2} \Sigma_{g}^{+}\right)$.

$V^{-}(R)$, along with the potential curve of the $X^{1} \Sigma_{g}^{+}$state of $\mathrm{Li}_{2}$, is shown. The potential minima of the $X$ and the $A$ curves are at 5.05 and 5.91 a.u., respectively. The two curves cross at $R=R_{s}=6.51$ a.u. and, therefore, the possibility of autodetachment of the resonant molecular anion exists only for internuclear separations smaller than $\boldsymbol{R}_{s}$, the so-called stabilization radius. The width (or, equivalently, the lifetime) $\Gamma(R)$ of this resonance is related to the imaginary part of the complex potential-energy curve of the $A^{2} \Sigma_{g}^{+}$state of $\mathrm{Li}_{2}{ }^{-}$. In the present autodetachment process ${ }^{2} \Sigma_{g}^{+} \rightarrow{ }^{1} \Sigma_{g}^{+}+e^{-}$, the lowest contributing partial wave is an $s$ wave and, thus, by Wigner's threshold law ${ }^{10}$ the width of this state is given by $\Gamma(R)=c k(R)$, where $k(R)$ is the wave number of the electron emitted at internuclear separation $R$ and $c$ is a constant. This constant $c$ is obtained as follows. The autodetachment width $\Gamma$ is related, by Fermi's golden rule, to the matrix element $V(R)$ coupling the discrete resonant state with the continuum state of the electronmolecule system. ${ }^{11}$ The wave function of the discrete $A^{2} \Sigma_{g}^{+}$state was estimated by smoothly extrapolating the fully optimized exponents of the CI wave functions from the variationally stable region $\left(R \geq R_{s}\right)$ into the autodetaching region $\left(R<R_{s}\right)$. The continuum state was approximated by extrapolation of a series of wave functions constructed by adding an electron, in a series of diffuse STO's, to the unperturbed ground-state wave function for $\mathrm{Li}_{2}\left(X^{1} \Sigma_{g}^{+}\right)$. The coupling matrix element $V(R)$ was then calculated by using these two wave functions. A comparison of the width calculated by using the golden rule with the threshold law expression, given above, yielded $c=0.0143$ a.u. The width of the $A^{2} \Sigma_{g}^{+}$state of $\mathrm{Li}_{2}{ }^{-}$as a function of the internuclear separation $R$ is shown in Fig. 2 and is given, in atomic units, by $\Gamma(R)$

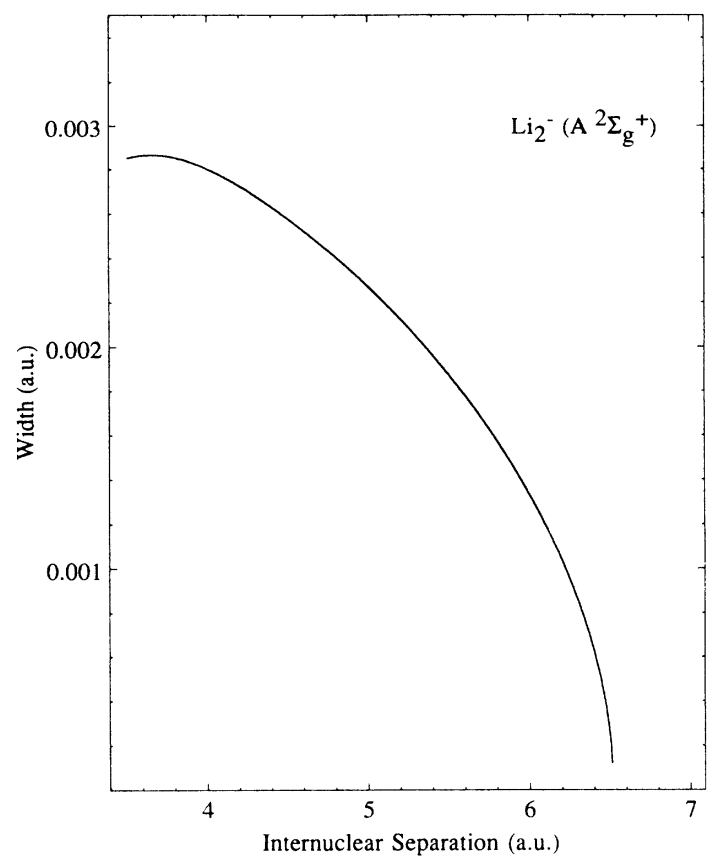

FIG. 2. The width of the $A^{2} \Sigma_{g}^{+}$state of $\mathrm{Li}_{2}{ }^{-}$.

$=0.0143 k(R)$.

In the traditional resonance theory, within the local approximation, the radial nuclear wave function $\xi(R)$ of the resonant anion state satisfies ${ }^{12}$

$$
\begin{aligned}
\int-\frac{\hbar^{2}}{2 M} \frac{d^{2}}{d R^{2}} & +\frac{\hbar^{2} J_{i}\left(J_{i}+1\right)}{2 M R^{2}}+V^{-}(R) \\
& \left.-\frac{1}{2} i \Gamma(R)-E\right] \xi(R)=-V(R) \zeta_{v_{i} J_{i}}(R),
\end{aligned}
$$

where $\zeta_{v_{i} J_{i}}(R)$ is the nuclear wave function of the initial rovibrational state of the neutral molecule and $M$ is the reduced mass of the nuclei. $E$ is the total energy of the system and its conservation in the initial channel (electron plus molecule) and the final channel (atom plus negative ion) provides an expression for the threshold for dissociative electron attachment (DA) to the molecule that is rovibrationally excited to a particular level,

$$
E_{\mathrm{th}}^{\mathrm{DA}}=\left\{\begin{array}{l}
D-N_{\mathrm{EA}}-E_{v J} \text { if } E_{v J}<D-N_{\mathrm{EA}}, \\
0, \text { otherwise. }
\end{array}\right.
$$

Here $D$ is the dissociation energy of the lithium molecule $(1.0372 \mathrm{eV}), N_{\mathrm{EA}}$ is the electron affinity of the lithium atom $(0.6182 \mathrm{eV})$ and $E_{v J}$ is the excitation energy of the initial rovibrational level of the molecule.

The nuclear wave function $\xi(R)$ is obtained by numerically solving Eq. (1) subject to the boundary conditions

$$
\begin{aligned}
& \xi(R=0)=0, \\
& \xi(R \rightarrow \infty) \rightarrow K R h_{J_{i}}^{(1)}(K R) .
\end{aligned}
$$

Here $\hbar^{2} K^{2} / 2 M$ is the relative kinetic energy of the ionatom pair after dissociative electron attachment and $h_{J_{i}}^{(1)}$ 
is the spherical Hankel function of the first kind.

If, after the formation of the temporary resonant state, the nuclei separate to $R>R_{s}$ without autodetachment having occurred, the detachment of the electron becomes energetically impossible and dissociative attachment can result. Thus for $R<R_{s}$, the envelope of $|\xi(R)|^{2} \mathrm{de}$ creases with $R$ because of the possibility of autodetachment and it is the asymptotic $(R \rightarrow \infty)$ value of $|\xi(R)|^{2}$ which determines the probability of dissociative electron attachment to the molecule. In fact, the integrated cross section for dissociative attachment of an electron with energy $\hbar^{2} k_{i}^{2} / 2 m$ to the molecule is given by ${ }^{12}$ (assuming momentum-normalized functions)

$$
\sigma_{\mathrm{DA}}=4 \pi^{2} \frac{m}{M} \frac{K}{k_{i}} \lim _{R \rightarrow \infty}|\xi(R)|^{2} .
$$

In order to convert the attachment cross sections into attachment rates one needs the energy distribution of electrons. In the present work we take this distribution to be Maxwellian, namely,

$$
f(E)=\left(\frac{27 E}{2 \pi \bar{E}^{3}}\right)^{1 / 2} \exp \left(-\frac{3 E}{2 \bar{E}}\right),
$$

where the average energy $\bar{E}$ is related to the electron temperature $T$ via $\bar{E}=3 k_{B} T / 2$. The attachment rate $k(\bar{E})$ is merely a convolution of $(2 E / m)^{1 / 2} \sigma_{\mathrm{DA}}(E)$, that is,

$$
k(\bar{E})=\left(\frac{2}{m}\right)^{1 / 2} \int_{0}^{\infty} E^{1 / 2} \sigma_{\mathrm{DA}}(E) f(E) d E .
$$

\section{RESULTS AND DISCUSSION}

In the present work we have calculated the cross sections and the rates for dissociative electron attachment to molecular lithium when the molecule is either in one of the vibrational levels $v=0$ to 12 , all rotationless, or in one of the rotational levels $J=0$ to 25 , all with $v=0$. Figure 3 shows the cross sections, as a function of the incident electron energy, for electron attachment to $\mathrm{Li}_{2}$ in various $(v, J)$ levels. Besides the lowest level $(0,0)$, the other two levels shown in Fig. $3(1,0)$ and $(0,22)$ have approximately the same internal energy. The attachment cross sections exhibit a rapid increase at the threshold, attaining a peak value $\sigma_{\text {peak }}$, followed by a uniform decrease as the energy of the incident electron increases. This almost vertical onset of the cross section is attributed to the attractive nature of the potential curve of the resonant anion state. ${ }^{13}$ The attachment rate is essentially determined by the peak attachment cross section. The results in Fig. 3 clearly show that the attachment cross section is enhanced if the molecular lithium is initially rovibrationally excited. A part of the enhancement of the cross section occurs due to the lowering of the threshold for attachment as the molecule is rovibrationally excited. Furthermore, for a fixed internal energy, vibrational excitation of the molecule is more effective in enhancing the cross section than rotational excitation. For example, when the molecule is initially provided with an internal energy of $0.04 \mathrm{eV}$, then the peak attachment cross section

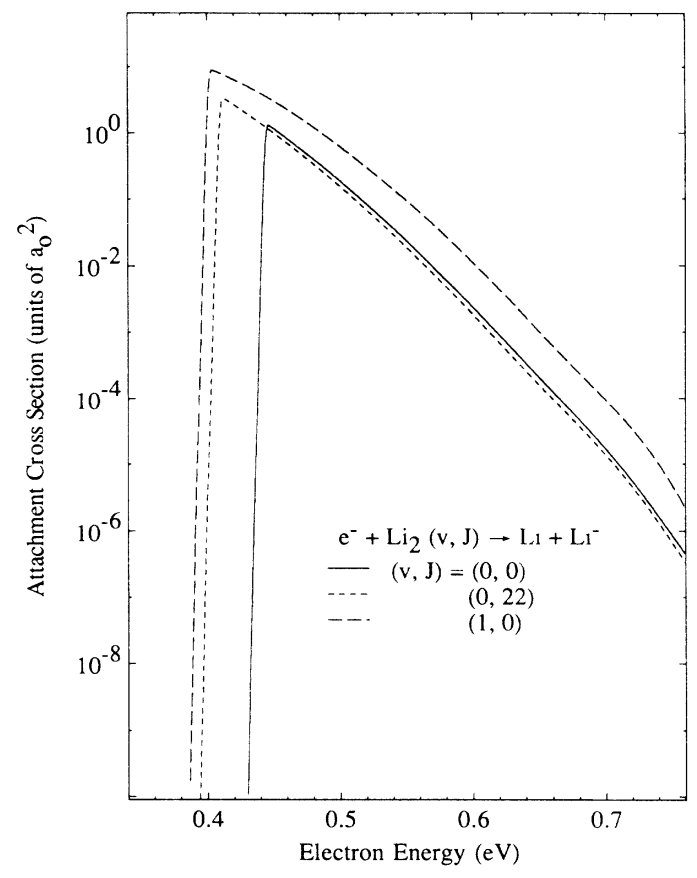

FIG. 3. The cross sections for dissociative attachment of low-energy electrons to molecular lithium in various rovibrational $(v, J)$ levels.

is increased by a factor of 6.8 if this internal energy is purely vibrational (from $v=0$ to 1 ) and by a factor of 2.5 if the internal energy is purely rotational (from $J=0$ to 22). Table I provides the energetics as well as the peak cross sections for dissociative electron attachment to the ground electronic state of molecular lithium in various

TABLE I. Internal rovibrational energy $\left(E_{v J}\right)$, threshold for dissociative attachment $\left(E_{\mathrm{th}}^{\mathrm{DA}}\right)$ and peak attachment cross section $\left(\sigma_{\text {peak }}\right)$ for various rovibrational levels of the ground electronic state of $\mathrm{Li}_{2}$. Asterisk denotes exoergic.

\begin{tabular}{rrlcc}
\hline \hline$v$ & $J$ & $E_{v J}(\mathrm{eV})$ & $E_{\mathrm{th}}^{\mathrm{DA}}(\mathrm{eV})$ & $\sigma_{\text {peak }}\left(\AA^{2}\right)$ \\
\hline 0 & 0 & 0.0 & 0.4190 & 0.368 \\
1 & 0 & 0.04292 & 0.3761 & 2.50 \\
2 & 0 & 0.08514 & 0.3339 & 3.96 \\
3 & 0 & 0.1267 & 0.2923 & 3.69 \\
4 & 0 & 0.1676 & 0.2514 & 4.52 \\
5 & 0 & 0.2078 & 0.2112 & 4.02 \\
6 & 0 & 0.2472 & 0.1718 & 9.26 \\
7 & 0 & 0.2860 & 0.1330 & 12.8 \\
8 & 0 & 0.3240 & 0.0950 & 12.8 \\
9 & 0 & 0.3612 & 0.0578 & 8.94 \\
10 & 0 & 0.3976 & 0.0214 & 10.3 \\
11 & 0 & 0.4333 & $*$ & 9.43 \\
0 & 1 & 0.00017 & 0.4188 & 0.369 \\
0 & 2 & 0.00050 & 0.4185 & 0.372 \\
0 & 5 & 0.00249 & 0.4165 & 0.389 \\
0 & 10 & 0.00910 & 0.4099 & 0.450 \\
0 & 20 & 0.03461 & 0.3844 & 0.809 \\
0 & 22 & 0.04164 & 0.3774 & 0.930 \\
0 & 25 & 0.05338 & 0.3656 & 1.16 \\
\hline \hline
\end{tabular}


rovibrational levels. It is interesting that, unlike molecular hydrogen, the peak attachment cross section here does not increase uniformly as the internal vibrational energy is increased. Whether this is due to the local nature of our present calculations or to the nature of the potential curves of the lithium molecule remains to be ascertained in the future. However, to maintain numerical consistency of our calculations the following equality was satisfied, as a check, to within a few parts in $10^{4}$ for all values of the incident electron energy and for all rovibrational levels of the molecule,

$$
\begin{aligned}
\frac{\hbar^{2} K}{M} \lim _{R \rightarrow \infty}|\xi(R)|^{2} & +\int_{0}^{\infty} \Gamma(R)|\xi(R)|^{2} d R \\
& =2 \int_{0}^{\infty} \operatorname{Im}\left[\xi^{*} V(R) \zeta_{v_{t} J_{l}}(R)\right] d R .
\end{aligned}
$$

This relationship is obtained by multiplying Eq. (1) by $\xi^{*}(R)$, subtracting the resulting equation from its complex conjugate and then integrating over $R$ from 0 to $\infty$.

Unlike the case of molecular hydrogen, the width of the resonance responsible for attachment to $\mathrm{Li}_{2}$ is quite small (or, equivalently, the lifetime is large) so that the resonance model is very reasonable. The enhancement of the attachment cross section is directly related to the increment of the range of internuclear separations, due to internal rovibrational excitation, over which the electron can be captured. This range is increased due to an increased vibrational amplitude during vibrational excitation and centrifugal stretching during rotational excitation. The probability of an electron capture, to form the resonant anion state, is maximum at an internuclear separation at which the energy difference between the potential curves of $\mathrm{Li}_{2}$ and $\mathrm{Li}_{2}{ }^{-}$is equal to the energy of the incident electron. This internuclear separation is referred to the capture radius, $R_{c}$. As the nuclei separate from $R_{c}$ to $R_{s}$ the autodetachment of the electron from the anion state, leaving behind a rovibrationally excited neutral molecule, is a distinct possibility. Dissociative attachment, of course, results when the internuclear separation far exceeds the stabilization radius $R_{s}$. For $\mathrm{Li}_{2}$ molecules in levels $v \geq 7$ with $J=0$ the range of intermolecular separations over which the electron could be captured includes $R_{s}$. The energetic threshold for dissociative electron attachment to $\mathrm{Li}_{2}$ is nonzero for vibrational levels $v \leq 10$. If the molecule is initially in vibrational levels $v \geq 11$, the attachment process is exoergic.

Figure 4 shows the effect of initial vibrational versus rotational excitation of the molecule on the rate of electron attachment as a function of electron temperature. As seen in this figure, the initial vibrational excitation to the $v=1(J=0)$ level enhances the attachment rate more dramatically than the initial rotational excitation to the $J=22(v=0)$ level which has roughly the same internal energy. The rate of electron attachment to $\mathrm{Li}_{2}$ in various vibrational levels is shown in Fig. 5. We note the interesting feature that the attachment rate saturates at the value $1.25 \times 10^{-8} \mathrm{~cm}^{3} \mathrm{~s}^{-1}$ which is reached when the molecule $\mathrm{Li}_{2}$ is initially in vibrational levels $v \sim 8-12$. Furthermore, this rate is achieved for electrons with an average energy of $\sim 0.2 \mathrm{eV}$. These results are consistent

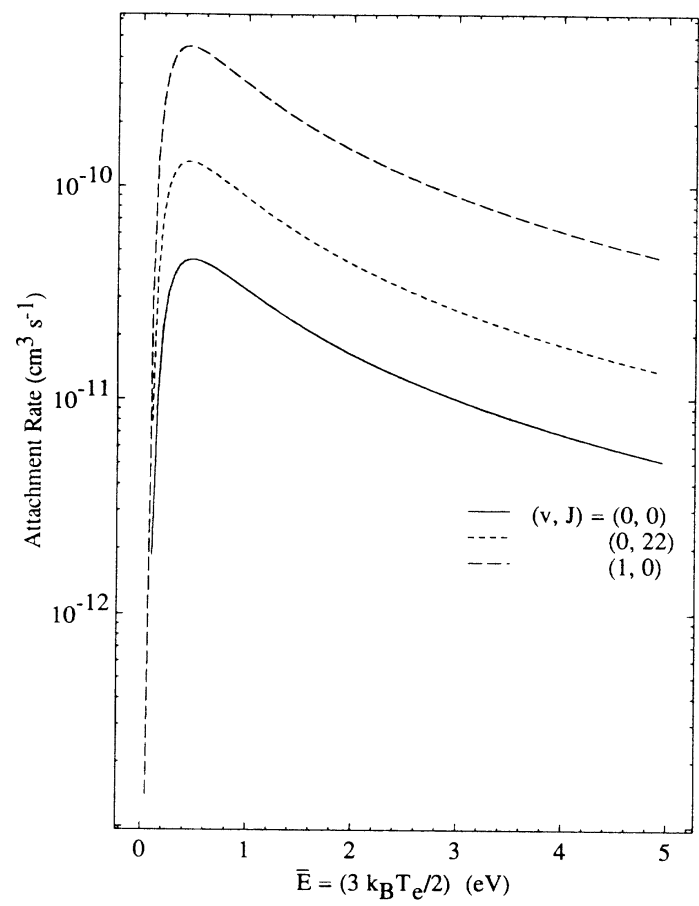

FIG. 4. Rates of dissociative electron attachment to $\mathrm{Li}_{2}$ in various rovibrational $(v, J)$ levels as a function of the electron temperature. The levels $(1,0)$ and $(0,22)$ have approximately the same excitation energy.

with the recent experimental observations ${ }^{3}$ of the rate of attachment of thermal electrons to molecular lithium.

A quantity which often is of interest to experimentalists and is useful for plasma diagnostic purposes is the en-

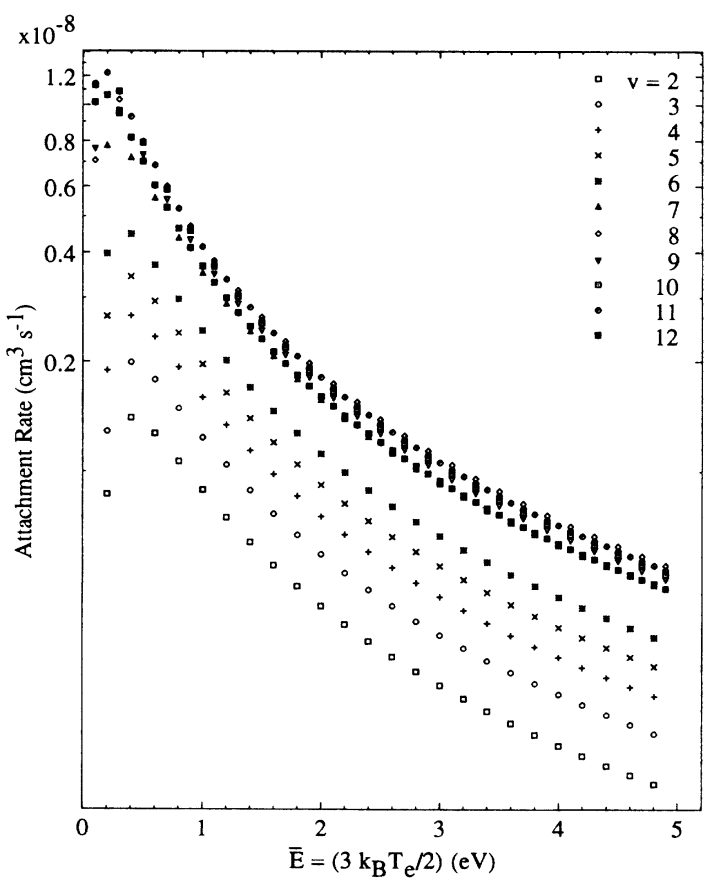

FIG. 5. Rates of dissociative electron attachment to $\mathrm{Li}_{2}$ in various vibrational levels as a function of the electron temperature. 
ergy of the negative ion formed during the process of dissociative electron attachment. The energy of this ion depends on the energy of the incident electron $E_{e}$ as well as on the excitation energy $E_{v J}$ of the initial rovibrational level of the molecule. In case of a homonuclear diatomic molecule like $\mathrm{Li}_{2}$ the atomic anion carries one-half of the relative kinetic energy of the ion-atom pair. The kinetic energy of the anion is

$$
E^{-}=\frac{1}{2}\left(E_{e}-D+N_{\mathrm{EA}}+E_{v J}\right) .
$$

Assuming a Maxwellian distribution of energies for the electrons, the average kinetic energy of the negative ions is given by

$$
\left\langle E^{-}\right\rangle=\int_{0}^{\infty} E^{-} \sigma_{\mathrm{DA}}(E) f(E) d E / \int_{0}^{\infty} \sigma_{\mathrm{DA}}(E) f(E) d E .
$$

Figure 6 shows, as a function of the electron temperature, the average energy of the $\mathrm{Li}^{-}$ions formed by dissociative electron attachment to $\mathbf{L i}_{2}$ in various vibrational levels. Since the attachment process becomes exoergic when the molecule is initially in vibrational levels $v \geq 11$, the relative kinetic energy of the ion-atom pair, then, is more than the energy of the incident electron and therefore the average energy of $\mathrm{Li}^{-}$ions formed by dissociative electron attachment to molecular lithium continues to increase as the internal vibrational energy increases. Among the vibrational levels that we have investigated $(v \sim 0-12)$ the maximum kinetic energy of the $\mathrm{Li}^{-}$ions is $0.153 \mathrm{eV}$.

In an actual plasma, though, the energy distribution of electrons is non-Maxwellian and only a detailed solution of the Boltzmann equation ${ }^{14}$ including all possible collision mechanisms of electrons provides the real distribution. If available, this real energy distribution of electrons should be used for calculating the attachment rates and the average energy of the negative ion.

We conclude by noting that the limited experimental information ${ }^{3}$ on the rates of dissociative electron attachment to molecular lithium that is currently available is consistent with our theoretical results. Detailed experiments investigating the effect of initial rovibrational exci-

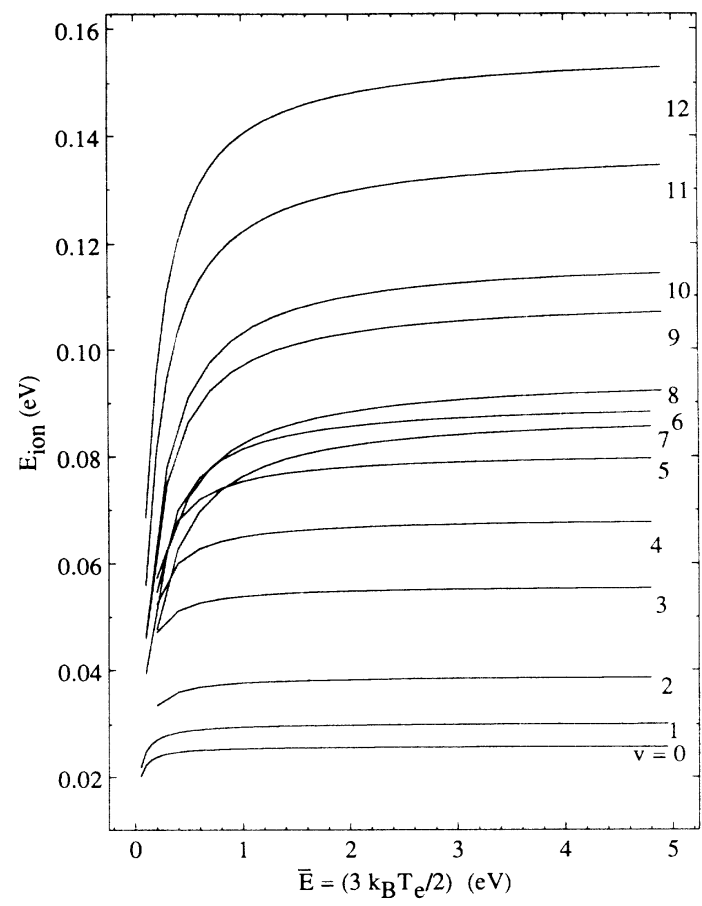

FIG. 6. Average energy of $\mathrm{Li}^{-}$ions formed by dissociative electron attachment to $\mathbf{L i}_{2}$ in various vibrational levels as a function of electron temperature.

tation of $\mathrm{Li}_{2}$ on the cross sections for formation of $\mathrm{Li}^{-}$ would be worthwhile in the future.

\section{ACKNOWLEDGMENTS}

It is a pleasure to thank Dr. H. H. Michels for providing the potential curves of $\mathrm{Li}_{2}$ and $\mathrm{Li}_{2}{ }^{-}$in numerical form and Dr. A. Garscadden for providing the conducive atmosphere of the Wright Research and Development Center where much of this work was done and written. The support of the U.S. Air Force Office of Scientific Research through Grant No. AFOSR-87-0342 is gratefully acknowledged.

*Permanent address.

1J. M. Wadehra and J. N. Bardsley, Phys. Rev. Lett. 41, 1795 (1978).

${ }^{2}$ M. Allan and S. F. Wong, Phys. Rev. Lett. 41, 1791 (1978).

${ }^{3}$ M. W. McGeoch and R. E. Schlier, Phys. Rev. A 33, 1708 (1986).

4J. M. Wadehra, Phys. Rev. A 29, 106 (1984).

${ }^{5}$ P. Kusch and M. M. Hessel, J. Chem. Phys. 67, 586 (1977); M. M. Hessel and C. R. Vidal, ibid. 70, 4439 (1979).

${ }^{6}$ H. Partridge, C. W. Bauschlicher, Jr., and P. E. M. Siegbahn, Chem. Phys. Lett. 97, 198 (1983).

${ }^{7}$ D. D. Konowalow and J. L. Fish, Chem. Phys. 77, 435 (1983);

Chem. Phys. Lett. 104, 210 (1984).

${ }^{8}$ K. K. Sunil and K. D. Jordan, Chem. Phys. Lett. 104, 343 (1984).

${ }^{9}$ H. H. Michels, R. H. Hobbs, and L. A. Wright, Chem. Phys. Lett. 118, 67 (1985).

${ }^{10}$ E. P. Wigner, Phys. Rev. 73, 1002 (1948).

${ }^{11}$ U. Fano, Phys. Rev. 124, 1866 (1961).

${ }^{12}$ J. M. Wadehra, in Nonequilibrium Vibrational Kinetics, edited by M. Capitelli (Springer-Verlag, Heidelberg, 1986), p. 191.

${ }^{13}$ T. F. O'Malley, Phys. Rev. 150, 14 (1966).

${ }^{14}$ P. J. Drallos and J. M. Wadehra, J. Appl. Phys. 63, 5601 (1988); Phys. Rev. A 40, 1967 (1989). 